MATHEMATICS OF COMPUTATION

Volume 66, Number 219, July 1997, Pages 1101-1117

S $0025-5718(97) 00847-8$

\title{
WAVEFORM RELAXATION AS A DYNAMICAL SYSTEM
}

\author{
MORTEN BJØRHUS AND ANDREW M. STUART
}

\begin{abstract}
In this paper the properties of waveform relaxation are studied when applied to the dynamical system generated by an autonomous ordinary differential equation. In particular, the effect of the waveform relaxation on the invariant sets of the flow is analysed. Windowed waveform relaxation is studied, whereby the iterative technique is applied on successive time intervals of length $T$ and a fixed, finite, number of iterations taken on each window. This process does not generate a dynamical system on $\mathbb{R}^{+}$since two different applications of the waveform algorithm over different time intervals do not, in general, commute. In order to generate a dynamical system it is necessary to consider the time $T$ map generated by the relaxation process. This is done, and $C^{1}$-closeness of the resulting map to the time $T$ map of the underlying ordinary differential equation is established. Using this, various results from the theory of dynamical systems are applied, and the results discussed.
\end{abstract}

\section{INTRODUCTION}

Consider the waveform relaxation (or dynamic iteration) scheme

$$
\left\{\begin{array}{c}
\text { For each } \ell=1,2,3, \ldots \text { find } u^{(\ell)}: \mathbb{R}^{+} \rightarrow \mathbb{R}^{m} \text { satisfying } \\
\dot{u}^{(\ell)}=\tilde{f}\left(u^{(\ell)}, u^{(\ell-1)}\right), u^{(\ell)}(0)=U \\
u^{(0)}(t) \equiv U
\end{array}\right.
$$

used to approach the solution $u: \mathbb{R}^{+} \rightarrow \mathbb{R}^{m}$ of the initial value problem

$$
\dot{u}=f(u), u(0)=U \text {. }
$$

Here $\tilde{f}(v, w)$, the splitting of $f$, must satisfy $\tilde{f}(u, u)=f(u)$ for all $u \in \mathbb{R}^{m}$ for consistency. The special splitting $\tilde{f}(v, w):=f(w)$ yields the classical Picard iteration.

Several papers have been written on the mathematical and numerical properties of this and related methods during the last decade; from the first article by Lelarasmee et al. [7], motivated by applications in electrical engineering, to the much referenced article by Miekkala and Nevanlinna [8], and later works by, for example, Nevanlinna [9], Bellen and Zennaro [1], and Bjørhus [2]. Much of the interest in the technique is motivated by the possibility of using it to exploit parallelism in software for initial value problems.

Under appropriate global Lipschitz conditions on $\tilde{f}$, the convergence of (1) to the solution of (2) is superlinear on any finite time interval. However the convergence

Received by the editor December 19, 1994 and, in revised form, October 16, 1995

1991 Mathematics Subject Classification. Primary 65L05, 34C35, 65Q05.

The first author was supported by the Research Council of Norway.

The second author was supported by the National Science Foundation and the Office for Naval Research. 
may be slow initially and so, instead of performing the waveform relaxation on the entire time interval of interest, one often confines the iteration to windows. That is, we choose $T>0$ and iterate successively on intervals of length $T$, starting with the interval $[0, T]$. In a practical implementation it is necessary to stop the iteration in a given window after a finite number of iterations, and then move on to the next window. We consider iterating a fixed number of times in each window. It should be emphasised, however, that the strategy we study here is far cruder than what is used in practice. Typically both the window size and the number of iterations is varied adaptively. The analysis we give is prototypical, playing the same role that analysis of fixed-step numerical methods for IVPs does in understanding adaptive software.

Define the $n$th window $I_{n}=(n T, n T+T)$ and let $k$ denote the fixed number of iterations taken in each time window. Let $u_{n}^{(\ell)}: \overline{I_{n}} \rightarrow \mathbb{R}^{m}$ denote the waveform with iterate number $\ell$ on the $n$th window. With this windowing strategy, our waveform relaxation scheme becomes, given $u_{-1}^{(k)}(0)=U$, the following:

$$
\left\{\begin{array}{l}
\text { For } n=0,1,2, \ldots \text { find } u_{n}^{(k)} \text { by solving } \\
\dot{u}_{n}^{(\ell)}=\tilde{f}\left(u_{n}^{(\ell)}, u_{n}^{(\ell-1)}\right) \text { in } I_{n}, u_{n}^{(\ell)}(n T)=u_{n-1}^{(k)}(n T), \\
\quad u_{n}^{(0)}(t) \equiv u_{n-1}^{(k)}(n T) \text { in } I_{n} \\
\text { for } \ell=1,2, \ldots, k
\end{array}\right.
$$

Let $\mathcal{S}(t), t \geq 0$, be the (generally nonlinear) semigroup of mappings from $\mathbb{R}^{m}$ to $\mathbb{R}^{m}$ corresponding to the solution of (2), so that

$$
\mathcal{S}(t) U=u(t) \quad \text { for } \quad 0 \leq t<\infty .
$$

If we define a similar family of mappings $\mathcal{S}_{k}(t)$ corresponding to our approximation (3), so that

$$
\mathcal{S}_{k}(t) U=u_{[t / T]}^{(k)}(t) \quad \text { for } 0 \leq t<\infty,
$$

where $[\cdot]$ denotes the integer part, it is easily seen that this family is in general not a semigroup. For example, take the Picard splitting with $k=1$. We then have

$$
\mathcal{S}_{1}(t) U=U+\int_{0}^{t} f(U) d \tau \text { for } 0 \leq t<T
$$

which is equivalent to approximating $u(t)$ using Euler's method. From (5) and the definition (4) of $\mathcal{S}_{1}(t)$ we have

$$
\begin{aligned}
\mathcal{S}_{1}(3 T / 2) U & =\mathcal{S}_{1}(T / 2) \mathcal{S}_{1}(T) U \\
& =(U+T f(U))+\int_{0}^{T / 2} f(U+T f(U)) d \tau \\
& =U+T f(U)+\frac{T}{2} f(U+T f(U)) .
\end{aligned}
$$


On the other hand, we have

$$
\begin{aligned}
\mathcal{S}_{1}(T) \mathcal{S}_{1}(T / 2) U & =\mathcal{S}_{1}(T)\left(U+\frac{T}{2} f(U)\right) \\
& =U+\frac{T}{2} f(U)+\int_{0}^{T} f\left(U+\frac{T}{2} f(U)\right) d \tau \\
& =U+\frac{T}{2} f(U)+T f\left(U+\frac{T}{2} f(U)\right) .
\end{aligned}
$$

Thus $\mathcal{S}_{1}(T) \mathcal{S}_{1}(T / 2) \neq \mathcal{S}_{1}(3 T / 2)$. This reflects the well-known fact that the mappings associated with Euler's method for two different steps do not commute. Hence the family $\left\{\mathcal{S}_{k}(t)\right\}$ cannot in general be a semigroup.

Our objective here is to examine the effect of the approximation (3) on the dynamical system generated by (2). Since the mapping $\mathcal{S}_{k}(t)$ does not define a dynamical system we are immediately faced with a conceptual problem. With this in mind we choose to formulate the problem in a different way, considering only the values of $u$ and $u_{n}^{(k)}$ at the points $n T, n=0,1,2, \ldots$ Thus, we only deal with the two sequences

$$
\{U, u(T), u(2 T), \ldots\} \text { and }\left\{U, u_{0}^{(k)}(T), u_{1}^{(k)}(2 T), \ldots\right\} .
$$

Let $S^{n}, n \geq 0$, be the discrete semigroup of mappings from $\mathbb{R}^{m}$ to $\mathbb{R}^{m}$ defined by $S^{n}=\mathcal{S}(n T)$; that is

$$
S^{n} U=u(n T) \text { for } n=0,1,2, \ldots \text {. }
$$

The semigroup property implies that the rule $S^{n+m}=S^{n} S^{m}=S^{m} S^{n}$ holds for all $n, m \geq 0$, and that $S^{0}=I$. Similarly, for our waveform relaxation scheme (3), we define the family of mappings $S_{k}^{n}$ as the mappings taking $U$ to the approximation to the solution at time $n T$; thus

$$
S_{k}^{n} U=u_{n-1}^{(k)}(n T) \text { for } n=0,1,2, \ldots .
$$

Now $S_{k}^{n}, n \geq 0$, is a semigroup, since it may be verified that $S_{k}^{0}=I$ and $S_{k}^{n+m}=$ $S_{k}^{n} S_{k}^{m}=S_{k}^{m} S_{k}^{n}$ for $n, m \geq 0$.

Throughout the paper we use $\|\cdot\|$ to denote the Euclidean norm on $\mathbb{R}^{m}$ (in Lemma 2.3 on $\left.\mathbb{R}^{2 m}\right)$ and also the induced operator norm on matrices in $L\left(\mathbb{R}^{m}, \mathbb{R}^{m}\right)$. By $B(x, r)$ we denote the closed ball in $\mathbb{R}^{m}$ centered at $x$ with radius $r$. For $g: \mathbb{R}^{m} \rightarrow \mathbb{R}^{m}$ we let $D g(x) \in L\left(\mathbb{R}^{m}, \mathbb{R}^{m}\right)$ denote the Fréchet derivative of $g$ at $x$. Similarly, for $g: \mathbb{R}^{m} \times \mathbb{R}^{m} \rightarrow \mathbb{R}^{m}$ we let $D_{i} g$ denote the derivative with respect to component $i, i=1,2$. We make the following simplifying assumptions at various points throughout the paper:

Assumption 1.1. The vector fields $f \in C^{1}\left(\mathbb{R}^{m}, \mathbb{R}^{m}\right)$ and $\tilde{f} \in C^{1}\left(\mathbb{R}^{m} \times \mathbb{R}^{m}, \mathbb{R}^{m}\right)$ are globally Lipschitz continuous, that is, there exist constants $K, K_{1}$, and $K_{2}>0$ such that, for all $x, y, v, w \in \mathbb{R}^{m}$,

$$
\begin{aligned}
\|f(v)-f(w)\| & \leq K\|v-w\|, \\
\|\tilde{f}(x, v)-\tilde{f}(y, w)\| & \leq K_{1}\|x-y\|+K_{2}\|v-w\| ;
\end{aligned}
$$

furthermore, $\tilde{f}$ is a consistent splitting of $f$, that is,

$$
\tilde{f}(u, u)=f(u) \quad \forall u \in \mathbb{R}^{m} .
$$


Assumption 1.2. Assumption 1.1 holds and, furthermore, the first derivatives of $f$ and $\tilde{f}$ are globally Lipschitz continuous, that is, there exist constants $B, B_{I}$ and $B_{I I}$ such that, for all $x, y, z, x^{\prime}, y^{\prime}, z^{\prime} \in \mathbb{R}^{m}$,

$$
\begin{aligned}
\left\|D f(x) y-D f\left(x^{\prime}\right) y^{\prime}\right\| & \leq B\left(\left\|x-x^{\prime}\right\|+\left\|y-y^{\prime}\right\|\right), \\
\left\|D_{1} \tilde{f}(x, y) z-D_{1} \tilde{f}\left(x^{\prime}, y^{\prime}\right) z^{\prime}\right\| & \leq B_{I}\left(\left\|x-x^{\prime}\right\|+\left\|y-y^{\prime}\right\|+\left\|z-z^{\prime}\right\|\right), \\
\left\|D_{2} \tilde{f}(x, y) z-D_{2} \tilde{f}\left(x^{\prime}, y^{\prime}\right) z^{\prime}\right\| & \leq B_{I I}\left(\left\|x-x^{\prime}\right\|+\left\|y-y^{\prime}\right\|+\left\|z-z^{\prime}\right\|\right) .
\end{aligned}
$$

Of course, imposing such global Lipschitz bounds is an unnecessarily strong assumption which can be removed with a slightly more subtle analysis. In several places we will comment on extensions to the locally Lipschitz case. Furthermore, it would also be possible to study this problem under one-sided Lipschitz conditions on $f$ and $\tilde{f}(\cdot, v)$, and a Lipschitz condition on $\tilde{f}(u, \cdot)$. The key is to establish an appropriate analogue of Proposition 2.4.

In Section 2 we prove some basic approximation results, showing $C^{1}$-closeness of the maps $S^{n}$ and $S_{k}^{n}$ and using these results to study the difference between $u(t)$ and $u_{n}^{(k)}(t)$. In Sections 3 and 4 these results are used to study the effect of the waveform relaxation on equilibria, phase portraits, periodic solutions and strange attractors. Finally note that we do not explicitly consider the effect of numerical approximation on the relaxation (3). However, the effect of this additional approximation could be incorporated in the analysis without great difficulty and we comment on this briefly in Section 5 .

\section{Some Approximation Results}

In order to use certain results from dynamical systems theory in the remainder of the paper, it is useful to show that our discrete semigroups satisfy, under suitable assumptions, the following two conditions:

(i)

$$
\left\|S_{k}^{1} U-S^{1} U\right\| \rightarrow 0 \quad \text { as } \quad k \rightarrow \infty
$$

uniformly for $U$ in bounded sets;

(ii)

$$
\left\|D S_{k}^{1}(U)-D S^{1}(U)\right\| \rightarrow 0 \quad \text { as } \quad k \rightarrow \infty,
$$

uniformly for $U$ in bounded sets.

Property (i) (resp. (ii)) is referred to as $C^{0}$-closeness (resp. $C^{1}$-closeness) of the mappings $S_{k}^{1}$ and $S^{1}$.

As an immediate consequence of the $C^{0}$-closeness, we can, if $f$ is globally Lipschitz continuous with constant $K$, bound the error $\mathcal{E}_{k}^{n+1}:=S_{k}^{n+1} U-S^{n+1} U$ by

$$
\begin{aligned}
\left\|\mathcal{E}_{k}^{n+1}\right\| & =\left\|S_{k}^{n+1} U-S^{n+1} U\right\|=\left\|S_{k}^{1} S_{k}^{n} U-S^{1} S^{n} U\right\| \\
& \leq\left\|S_{k}^{1} S_{k}^{n} U-S^{1} S_{k}^{n} U\right\|+\left\|S^{1} S_{k}^{n} U-S^{1} S^{n} U\right\|
\end{aligned}
$$

so that, by continuity of the semigroup $\mathcal{S}(t)$,

$$
\left\|\mathcal{E}_{k}^{n+1}\right\| \leq\left\|S_{k}^{1} S_{k}^{n} U-S^{1} S_{k}^{n} U\right\|+e^{K T}\left\|\mathcal{E}_{k}^{n}\right\| .
$$

Successive application of this estimate, together with (i), will yield an estimate of $\left\|\mathcal{E}_{k}^{N}\right\|$. Hence we can analyze convergence properties of our iteration. We will give the precise result later in this section. The $C^{1}$-closeness will allow us to consider the effect for the approximation (3) on more complicated invariant sets of (2). 
We will need the following lemma, relating the iterates appearing in (1) to the solution of (2).

Lemma 2.1. Let Assumption 1.1 hold. For any $t>0$, let $u$ solve $(2)$ with $u(0)=U$ and let $v^{(k)}$ solve (1) with $v^{(k)}(0)=V$ and $v^{(0)}(s)=V, 0<s<t$. Then, if

$$
C_{1}(t)=\frac{1}{K} e^{\left(K_{1}+K\right) t}, \quad \varepsilon(k, t)=\frac{\left(K_{2} t\right)^{k}}{k !},
$$

it follows that

$$
\left\|v^{(k)}(t)-u(t)\right\| \leq C_{1}(t) \varepsilon(k, t)\|f(V)\|+e^{K t}\|U-V\| .
$$

Proof. Let $v$ be the solution of (2) with $v(0)=V$. We have

$$
\left\|v^{(k)}(t)-u(t)\right\| \leq\left\|v^{(k)}(t)-v(t)\right\|+\|v(t)-u(t)\| .
$$

The last quantity on the right satisfies the standard estimate

$$
\|v(t)-u(t)\| \leq e^{K t}\|V-U\| .
$$

It remains to estimate the quantity $\left\|v^{(k)}(t)-v(t)\right\|$. First we note that we have (on the open intervals where $\left.v^{(k)}(t) \neq v(t)\right)$ the differential inequality

$$
\frac{d}{d t}\left\|v^{(k)}(t)-v(t)\right\| \leq K_{1}\left\|v^{(k)}(t)-v(t)\right\|+K_{2}\left\|v^{(k-1)}(t)-v(t)\right\| .
$$

An application of Gronwall's lemma yields a bound for $\left\|v^{(k)}(t)-v(t)\right\|$ in terms of $K_{1}, K_{2}$, and $\left\|v^{(k-1)}(t)-v(t)\right\|$. Successive application of this bound yields an iterated integral which can be estimated crudely to give

$$
\left\|v^{(k)}(t)-v(t)\right\| \leq \varepsilon(k, t) e^{K_{1} t} \max _{0 \leq s \leq t}\left\|v^{(0)}(s)-v(s)\right\| ;
$$

see, for example, [2]. The last norm can be estimated, again by using Gronwall's lemma in a standard fashion, as

$$
\max _{0 \leq s \leq t}\left\|v^{(0)}(s)-v(s)\right\|=\max _{0 \leq s \leq t}\|V-v(s)\| \leq \frac{e^{K t}}{K}\|f(V)\|,
$$

and we thus obtain the estimate

$$
\left\|v^{(k)}(t)-v(t)\right\| \leq C_{1}(t) \varepsilon(k, t)\|f(V)\| .
$$

This completes the proof.

Remark 2.2. Although we have assumed globally Lipschitz $f$ and $\tilde{f}$, it is worth remarking that superlinear convergence can be established in the locally Lipschitz case in the following two situations: (a) if $T$ is sufficiently small, then the map from the function $u^{(\ell)}(t)$ to the function $u^{(\ell+1)}(t)$ takes a bounded set into itself and hence the globally Lipschitz result may be adapted to apply within this bounded set; (b) if the sequence of functions $\left\{u^{(\ell)}(t)\right\}_{\ell=0}^{\infty}$ is uniformly bounded in the supremum norm on $[0, T]$ then the globally Lipschitz result may be adapted to apply, on a set satisfying the appropriate bound, to deduce superlinear convergence of the iteration.

In the following, let $C_{1}^{\prime}(t)$ and $\varepsilon^{\prime}(k, T)$ be given by $C_{1}(t)$ and $\varepsilon(k, T)$ (see (11)) with $K \mapsto \sqrt{2}(B+K), K_{i} \mapsto \sqrt{2}\left(B_{I}+B_{I I}+K_{i}\right)$ for $i=1,2$. Now we are in the position to show the following result. 
Lemma 2.3. Let Assumption 1.2 hold. Then, for all $T>0$,

$$
\left\|S_{k}^{1} U-S^{1} U\right\|+\left\|D S_{k}^{1}(U)-D S^{1}(U)\right\| \leq \sqrt{2} \cdot C_{1}^{\prime}(T) \cdot \varepsilon^{\prime}(k, T)(\|f(U)\|+K)
$$

for all $U \in \mathbb{R}^{m}$.

Proof. In the following $D_{U}$ denotes differentiation with respect to $U$. Let $u(t)=$ $u(U, t)$, and define for $\ell=1,2, \ldots$ and for all $\xi \in \mathbb{R}^{m}$

$$
\eta_{\xi}(t)=\left(\begin{array}{c}
u(t) \\
D_{U} u(U, t) \xi
\end{array}\right), \eta_{\xi}^{(\ell)}(t)=\left(\begin{array}{c}
u^{(\ell)}(t) \\
D_{U} u^{(\ell)}(U, t) \xi
\end{array}\right) .
$$

We can see that $\eta_{\xi}$ and $\eta_{\xi}^{(\ell)}$ satisfy the initial value problems

$$
\dot{\eta}_{\xi}=g\left(\eta_{\xi}\right), \eta_{\xi}(0)=\left(\begin{array}{c}
U \\
\xi
\end{array}\right)
$$

and

$$
\dot{\eta}_{\xi}^{(\ell)}=\tilde{g}\left(\eta_{\xi}^{(\ell)}, \eta_{\xi}^{(\ell-1)}\right), \eta_{\xi}^{(\ell)}(0)=\left(\begin{array}{c}
U \\
\xi
\end{array}\right), \eta_{\xi}^{(0)}(t) \equiv\left(\begin{array}{c}
U \\
\xi
\end{array}\right) ;
$$

here the functions $g: \mathbb{R}^{2 m} \rightarrow \mathbb{R}^{2 m}$ and $\tilde{g}: \mathbb{R}^{2 m} \times \mathbb{R}^{2 m} \rightarrow \mathbb{R}^{2 m}$ are given by (for $x_{i}, y_{i} \in \mathbb{R}^{m}$ )

$$
g\left(\left[\begin{array}{l}
x_{1} \\
x_{2}
\end{array}\right]\right)=\left(\begin{array}{c}
f\left(x_{1}\right) \\
D f\left(x_{1}\right) x_{2}
\end{array}\right)
$$

and

$$
\tilde{g}\left(\left[\begin{array}{l}
x_{1} \\
x_{2}
\end{array}\right],\left[\begin{array}{l}
y_{1} \\
y_{2}
\end{array}\right]\right)=\left(\begin{array}{c}
\tilde{f}\left(x_{1}, y_{1}\right) \\
D_{1} \tilde{f}\left(x_{1}, y_{1}\right) x_{2}+D_{2} \tilde{f}\left(x_{1}, y_{1}\right) y_{2}
\end{array}\right) .
$$

Note that $\tilde{g}(\eta, \eta)=g(\eta)$ by Assumption 1.2. We see that the equations satisfied by $\eta_{\xi}$ and $\eta_{\xi}^{(\ell)}$ are completely analogous to equations (2) and (1), respectively. Recall that $\|\cdot\|$ defines the Euclidean norm on $\mathbb{R}^{2 m}$. Writing vectors $w \in \mathbb{R}^{2 m}$ in the form $w=\left(u^{T}, v^{T}\right)^{T}$, with $u, v \in \mathbb{R}^{m}$, we can define an equivalent norm $|\cdot|$ on $\mathbb{R}^{2 m}$ by

$$
|w|=\|u\|+\|v\|,
$$

satisfying

$$
\|w\| \leq|w| \leq \sqrt{2}\|w\|
$$

Using this it follows from Assumption 1.1 that $g$ and $\tilde{g}$ satisfy Lipschitz conditions analogous to (7) and (8) with $K \mapsto \sqrt{2}(B+K), K_{i} \mapsto \sqrt{2}\left(B_{I}+B_{I I}+K_{i}\right)$ for $i=1,2$. Thus we can verify the assumptions of Lemma 2.1 (for the case $V=U$ ), with $g, \tilde{g}, 2 m$ instead of $f, \tilde{f}, m$. We find that

$$
\left\|u^{(k)}(T)-u(T)\right\|+\left\|D_{U} u^{(k)}(U, T) \xi-D_{U} u(U, T) \xi\right\| \leq \sqrt{2} \cdot\left\|\eta_{\xi}^{(k)}(T)-\eta_{\xi}(T)\right\| .
$$

By Lemma 2.1 we know that this last norm is bounded by $C_{1}^{\prime}(T) \cdot \varepsilon^{\prime}(k, T) \cdot\left\|g\left(\eta_{\xi}(0)\right)\right\|$. But, by (13),

$$
\sqrt{2} \cdot C_{1}^{\prime}(T) \cdot \varepsilon^{\prime}(k, T) \cdot\left\|g\left(\eta_{\xi}(0)\right)\right\| \leq \sqrt{2} \cdot C_{1}^{\prime}(T) \cdot \varepsilon^{\prime}(k, T) \cdot(\|f(U)\|+\|D f(U) \xi\|) .
$$

Since $f$ is globally Lipschitz with constant $K$ so that $\|D f(U)\| \leq K$, this yields the estimate (12) after taking the supremum over $\|\xi\|=1$. The proof is complete.

The estimates we set out to establish now follow from Lemma 2.3: 
Proposition 2.4. Let Assumption 1.1 hold. Then there exists a constant $C_{2}=$ $C_{2}(T, R)$ such that, for all $U \in B(0, R)$ and all $T>0$,

$$
\left\|S_{k}^{1} U-S^{1} U\right\| \leq C_{2} \varepsilon(k, T) .
$$

Let Assumption 1.2 hold. Then there exists a constant $C_{3}=C_{3}(T, R)$ such that, for all $U \in B(0, R)$ and all $T>0$,

$$
\left\|D S_{k}^{1}(U)-D S^{1}(U)\right\| \leq C_{3} \varepsilon^{\prime}(k, T) .
$$

Proof. In fact, from Lemma 2.3, we see that for a given $U$ both quantities can be bounded by the same expression, namely

$$
\sqrt{2} \cdot C_{1}^{\prime}(T) \cdot \varepsilon^{\prime}(k, T)(\|f(U)\|+K) .
$$

However, $f(U)$ being a continuous function in $\mathbb{R}^{m}$ implies that its composition with a norm is continuous. Since a continuous function always attains its maximum on a compact set, we can find a constant $C_{f}(R)$ such that

$$
\sup _{U \in B(0, R)}\|f(U)\| \leq C_{f}(R) .
$$

Hence we get the estimate (15) by putting $C_{3}=\sqrt{2} \cdot C_{1}^{\prime}(T)\left(C_{f}(R)+K\right)$. Lemma 2.1 (with $V=U$ ) yields estimate (14) by letting $C_{2}(T, R)=C_{1}(T) \cdot C_{f}(R)$.

Now we can give the precise form of the convergence result promised at the beginning of this section.

Theorem 2.5. Let Assumption 1.1 hold and let $\delta>0$. Assume that $\left\|S^{n} U\right\| \leq r$ for all $0 \leq n \leq N$ and that $k$ is chosen so that

$$
\left(\frac{e^{N K T}-1}{e^{K T}-1}\right) C_{4} \varepsilon(k, T) \leq \delta,
$$

where $C_{4}=C_{2}(T, r+\delta)$. Then

$$
\left\|S_{k}^{n} U-S^{n} U\right\| \leq\left(\frac{e^{n K T}-1}{e^{K T}-1}\right) C_{4} \varepsilon(k, T) \quad \forall 0 \leq n \leq N .
$$

Proof. Assume for the purposes of induction that (16) holds for $n=m \leq N-1$, noting that this is true for $n=0$. By (16) it follows that

$$
\left\|S_{k}^{m} U-S^{m} U\right\| \leq\left(\frac{e^{N K T}-1}{e^{K T}-1}\right) C_{4} \varepsilon(k, T) \leq \delta
$$

so that $S_{k}^{m} U \in B(0, r+\delta)$. Thus, by (10), and (14),

$$
\begin{aligned}
\left\|\mathcal{E}_{k}^{m+1}\right\| & \leq C_{4} \varepsilon(k, T)+e^{K T}\left\|\mathcal{E}_{k}^{m}\right\| \\
& \leq C_{4} \varepsilon(k, T)+e^{K T}\left(\frac{e^{m K T}-1}{e^{K T}-1}\right) C_{4} \varepsilon(k, T) \\
& =\left(\frac{e^{(m+1) K T}-1}{e^{K T}-1}\right) C_{4} \varepsilon(k, T) .
\end{aligned}
$$

The result follows by induction.

Corollary 2.6. Let the assumptions of Theorem 2.5 hold, and let $t=n T+\tau$ where $0 \leq n \leq N$ and $\tau \in[0, T)$. Then there exists $C_{5}=C_{5}(T, r+\delta)$ such that

$$
\left\|u_{n}^{(k)}(t)-u(t)\right\| \leq C_{5} \varepsilon(k, T) .
$$


Proof. Applying Lemma 2.1 we have

$$
\begin{aligned}
\left\|u_{n}^{(k)}(t)-u(t)\right\| & \leq\left\|\mathcal{S}_{k}(t) U-\mathcal{S}(t) U\right\| \\
& =\left\|\mathcal{S}_{k}(\tau) S_{k}^{n} U-\mathcal{S}(\tau) S^{n} U\right\| \\
& \leq C_{1}(\tau) \varepsilon(k, \tau)\left\|f\left(S_{k}^{n} U\right)\right\|+e^{K \tau}\left\|S_{k}^{n} U-S^{n} U\right\| .
\end{aligned}
$$

Noting that $S_{k}^{n} U \in B(0, r+\delta)$ and that $\tau \leq T, C_{1}(\tau) \leq C_{1}(T), \varepsilon(k, \tau) \leq \varepsilon(k, T)$, applying Theorem 2.5 gives the desired result.

\section{NeIghbourhood OF AN EQUILIBRIUM POINT}

In this section we compare the behaviour of (2) and (3) in the neighbourhood of an equilibrium point of (2). We use the $C^{1}$-closeness established in Section 2 to apply some basic results from the theory of dynamical systems.

We make the following definitions:

$$
\begin{aligned}
\mathcal{E} & =\left\{v \in \mathbb{R}^{m} \mid \mathcal{S}(t) v \equiv v \forall t \geq 0\right\}, \\
\mathcal{E}_{k} & =\left\{v \in \mathbb{R}^{m} \mid \mathcal{S}_{k}(t) v \equiv v \forall t \geq 0\right\} .
\end{aligned}
$$

Thus $\mathcal{E}$ and $\mathcal{E}_{k}$ denote the equilibria of (2) and (3) respectively. We first show that $\mathcal{E} \equiv \mathcal{E}_{k}$ so that the waveform relaxation does not introduce spurious equilibria when viewed as a mapping on $\mathbb{R}^{+}$.

Theorem 3.1. For any vector fields $f$ and $\tilde{f}$ satisfying Assumption 1.1 it follows that $\mathcal{E} \equiv \mathcal{E}_{k}$.

Proof. First let $v \in \mathcal{E}$ so that $f(v)=0$. Since $\tilde{f}(u, u)=f(u)$ for all $u \in \mathbb{R}^{m}$, it follows that $\tilde{f}(v, v)=0$. Thus

$$
u_{n}^{(\ell)}(t) \equiv v, \quad \forall \ell=1,2, \ldots, k, \forall n=0,1,2, \ldots, \forall t \in \overline{I_{n}}
$$

is a solution of (3) with $U=v$ so that $v \in \mathcal{E}_{k}$ follows. Thus $\mathcal{E} \subseteq \mathcal{E}_{k}$.

Secondly, let $u_{n}^{(k)} \equiv v \in \mathcal{E}_{k}$. It follows that

$$
\dot{u}_{n}^{(k)}(t)=0 \quad \forall t \in I_{n} .
$$

From this we deduce that

$$
\tilde{f}\left(u_{n}^{(k)}(t), u_{n}^{(k-1)}(t)\right)=0 \quad \forall t \in I_{n} .
$$

Thus

$$
\tilde{f}\left(v, u_{n}^{(k-1)}(t)\right)=0 \quad \forall t \in I_{n} .
$$

Hence, since $u_{n}^{(\ell)}(t)$ is continuous in $t$ and since $f(v, \cdot)$ is a continuous function, it follows that

$$
\tilde{f}\left(v, u_{n}^{(k-1)}(n T)\right)=0 .
$$

But $u_{n}^{(\ell)}(t)=u_{n-1}^{(k)}(n T)=v$ for $\ell=1, \ldots, k$ since $v \in \mathcal{E}_{k}$. Thus we have

$$
\tilde{f}(v, v)=0 .
$$

Since $f(v)=\tilde{f}(v, v)$ we deduce that $v \in \mathcal{E}$ and hence that $\mathcal{E}_{k} \subseteq \mathcal{E}$. The proof is complete. 
Theorem 3.1 should be interpreted with caution: since the natural dynamical system for (3) is found by considering the time $T$ map, we are led to study, instead of (17), the fixed points of $S^{1}$ and $S_{k}^{1}$; thus we define

$$
\begin{aligned}
& \mathcal{E}^{*}=\left\{v \in \mathbb{R}^{m} \mid S^{1} v=v\right\}, \\
& \mathcal{E}_{k}^{*}=\left\{v \in \mathbb{R}^{m} \mid S_{k}^{1} v=v\right\} .
\end{aligned}
$$

As we shall see, these sets are not necessarily the same. In fact we prove:

Theorem 3.2. For any vector fields $f$ and $\tilde{f}$ satisfying Assumption 1.1 we have:

(i) $\mathcal{E}^{*} \supseteq \mathcal{E}$; if $v \in \mathcal{E}^{*}$ and $v$ is an isolated point in $\mathcal{E}^{*}$, then $v \in \mathcal{E}$.

(ii) $\mathcal{E}_{k}^{*} \supseteq \mathcal{E}$; if $v \in \mathcal{E}_{k}^{*}$ and $v$ is an isolated point in $\mathcal{E}_{k}^{*}$, then it is not necessarily the case that $v \in \mathcal{E}$.

Proof. Clearly $\mathcal{E}^{*} \supseteq \mathcal{E}$ since $\mathcal{E}^{*}$ comprises equilibria together with points on periodic solutions of period $T$. If $v \in \mathcal{E}^{*}$ is isolated, then it cannot be a point on a periodic solution. Hence $v \in \mathcal{E}$ and (i) follows.

Clearly $\mathcal{E}_{k}^{*} \supseteq \mathcal{E}_{k} \equiv \mathcal{E}$. Thus to complete the proof of (ii) we need only find a counterexample to illustrate the final point. Consider (ii) in the case $k=2$ and $\tilde{f}(u, v)=f(v)$. Then, if $w^{(\ell)}(t)=u_{0}^{(\ell)}(t)$ we have

$$
\frac{d w^{(\ell)}}{d t}(t)=f\left(w^{(\ell-1)}(t)\right), \quad w^{(\ell)}(0)=U,
$$

$w^{0}(t) \equiv U, t \in I_{1}$ and

$$
w^{(1)}(t)=U+t f(U)
$$

Thus

$$
w^{(2)}(t)=U+\int_{0}^{t} f(U+s f(U)) d s .
$$

Hence

$$
S_{2}^{1} U=U+\int_{0}^{T} f(U+s f(U)) d s .
$$

To find an isolated fixed point of $S_{2}^{1}: \mathbb{R}^{m} \rightarrow \mathbb{R}^{m}$, let $\rho=\sqrt{2 / T}$, choose a number $r>\rho$ satisfying $r>\max _{-\rho \leq x \leq \rho} \max _{0<s<T}\left(x+s x^{3}\right)$, and consider the function $f(x)=-\Phi_{r}(x) x^{3}$ where $\Phi_{r}(x) \in C^{\infty}(\mathbb{R}, \mathbb{R})$ satisfies $\Phi_{r}=1$ for $|x| \leq r$, and $\Phi_{r}=0$ for $|x| \geq 2 r$. Note that $f(x)$ is constructed to be globally Lipschitz but that it behaves like $x^{3}$ if $x$ is not large; this is the reason for the construction. Then, for $-\rho \leq U \leq \rho$ we have

$$
\begin{aligned}
S_{2}^{1} U & =U+\int_{0}^{T}-\left(U-s U^{3}\right)^{3} d s \\
& =U+\left[\frac{\left(U-s U^{3}\right)^{4}}{4 U^{3}}\right]_{0}^{T} \\
& =U+\frac{U}{4}\left(1-T U^{2}\right)^{4}-\frac{U}{4} .
\end{aligned}
$$

The fixed points of $S_{2}^{1}$ in $[-\rho, \rho]$ are thus

$$
U=0, \quad U=\rho=\sqrt{2 / T} .
$$


Clearly $0 \in \mathcal{E}$; but $\sqrt{2 / T} \notin \mathcal{E}$ and it is an isolated point in $\mathcal{E}_{k}^{*}$ so that (ii) follows.

Remark 3.3. The construction of the counterexample in proving (ii) of Theorem 3.2 is motivated by results concerning spurious fixed points of Runge-Kutta methods; see [6] for example.

As a consequence of Theorem 3.1 and Theorem 3.2 we now know that equilibria of (2) are fixed points of $S^{1}$ and $S_{k}^{1}$. It is natural to consider the behaviour of these maps in the neighbourhood of such fixed points. By doing this we can make deductions about the solutions of (2) and (3) near to equilibria.

Let the sequences $\left\{u_{n}\right\}_{n=0}^{\infty}$ and $\left\{U_{n}\right\}_{n=0}^{\infty}$ be generated by the maps

$$
u_{n+1}=S^{1} u_{n}
$$

and

$$
U_{n+1}=S_{k}^{1} U_{n}
$$

and assume that (2) has a hyperbolic equilibrium point $v$. Thus $v \in \mathcal{E}^{*}$ and, furthermore, $v$ is a hyperbolic fixed point of $S^{1}: \mathbb{R}^{m} \rightarrow \mathbb{R}^{m}$. This follows since

$$
L:=D S^{1}(v)=\exp (D f(v) T)
$$

and the hyperbolicity of $v$ as an equilibrium of (2) implies that no point of the spectrum of $D f(v)$ lies on the imaginary axis; it follows that no point of the spectrum of $L$ lies on the unit circle.

Let $\mathcal{P}$ and $\mathcal{Q}$ denote projections onto the subspaces $Y$ and $Z$ corresponding to spectrum of $L$ strictly outside and strictly inside the unit circle respectively. These projections are defined through the Jordan normal form of $L$ and the following properties hold (see [11], Appendix B for example): (i)

$$
Y=\mathcal{P} \mathbb{R}^{m}, \quad Z=\mathcal{Q} \mathbb{R}^{m}, \quad \mathbb{R}^{m}=Y \oplus Z ;
$$

(ii) there exists $a<1$ and norms $\|\cdot\|_{u},\|\cdot\|_{s}$ on $\mathbb{R}^{m}$ in which

$$
\begin{gathered}
\left\|L^{-1} y\right\|_{u} \leq a\|y\|_{u} \quad \forall y \in Y \\
\|L z\|_{s} \leq a\|z\|_{s} \quad \forall z \in Z ;
\end{gathered}
$$

(iii) if we define

$$
\|\cdot\|_{x}=\max \left\{\|\mathcal{P} \cdot\|_{u},\|\mathcal{Q} \cdot\|_{s}\right\}
$$

then this is equivalent to the Euclidean norm $\|\cdot\|$ on $\mathbb{R}^{m}$.

The local phase portrait of the mappings $S^{1}$ and $S_{k}^{1}$ may be found by solving (18), (19) subject to

$$
\begin{array}{ll}
\mathcal{P}\left[u_{N}-v\right]=\xi, & \mathcal{Q}\left[u_{0}-v\right]=\eta, \\
\mathcal{P}\left[U_{N}-v\right]=\xi, & \mathcal{Q}\left[U_{0}-v\right]=\eta .
\end{array}
$$

Applying Theorems 4.13 and 4.17 of [10], which require the $C^{1}$-closeness established in Proposition 2.4 under Assumption 1.2, we obtain the following result:

Theorem 3.4. Let Assumption 1.2 hold, let $v$ be a hyperbolic equilibrium point of (2) and let $\xi \in Y, \eta \in Z$ satisfy $\|\xi\|_{x},\|\eta\|_{x} \leq \delta$. Then there are constants $C_{6}, C_{7}>0$ 
such that, for all such $\xi$ and $\eta$, for all $\delta$ and $\varepsilon(k, T)$ sufficiently small, and all integers $N$, problems (18), (20) and (19), (21) have unique solutions satisfying

$$
\max _{0 \leq n \leq N}\left\|u_{n}-v\right\|_{x}, \max _{0 \leq n \leq N}\left\|U_{n}-v\right\|_{x} \leq C_{6} \delta
$$

furthermore,

$$
\max _{0 \leq n \leq N}\left\|u_{n}-U_{n}\right\|_{x} \leq C_{7} \varepsilon(k, T) .
$$

A straightforward corollary is the following:

Corollary 3.5. Let Assumption 1.2 hold, let $v$ be a hyperbolic equilibrium point of (2) and let $\xi \in Y$ and $\eta \in Z$ satisfy $\|\xi\|_{x},\|\eta\|_{x} \leq \delta$. Then there are constants $C_{8}, C_{9}>0$ such that, for all such $\xi$ and $\eta$, for all $\delta$ and $\varepsilon(k, T)$ sufficiently small, and all integers $N$, equations (2) and (3) have solutions satisfying

$$
\begin{array}{cc}
\mathcal{P}[u(N T)-v]=\xi, & \mathcal{Q}[u(0)-v]=\eta, \\
\mathcal{P}\left[u_{N-1}^{(k)}(N T)-v\right]=\xi, & \mathcal{Q}\left[u_{-1}^{(k)}(0)-v\right]=\eta ;
\end{array}
$$

furthermore,

$$
\max _{0 \leq t \leq N T}\|u(t)-v\|_{x}, \max _{0 \leq n \leq N-1} \max _{0 \leq t \leq T}\left\|u_{n}^{(k)}(n T+t)-v\right\|_{x} \leq C_{8} \delta
$$

and

$$
\max _{0 \leq n \leq N-1} \max _{0 \leq t \leq T}\left\|u(n T+t)-u_{n}^{(k)}(n T+t)\right\|_{x} \leq C_{9} \varepsilon(k, T) .
$$

Remark 3.6. Since $N$ is arbitrary, the previous result allows us to interpret certain solutions of (2) and (3) as being uniformly close, over arbitrarily long time intervals, in the neighbourhood of hyperbolic equilibria. This improves upon Corollary 2.6 which is not uniformly valid in time. The key to the improved result in the neighbourhood of equilibria is that the solutions of (2) and (3) have different initial conditions. Hence we have a shadowing-type result. Figure 1 illustrates three solutions of (2), with fixed $\xi$ and $\eta$, corresponding to three different values of $N$. Notice that, the larger $N$, the closer the solution lies to the equilibrium point $v$. In fact, as $N \rightarrow \infty$, the solution must approach arbitrarily close to $v$ at some point on its trajectory.

Next we turn our attention to unstable manifolds. In the following we will need the Hausdorff semi-distance $\operatorname{dist}(A, B)$ and the Hausdorff distance $d(A, B)$. Recall that

$$
\operatorname{dist}(A, B)=0 \Leftrightarrow \bar{A} \subseteq \bar{B}
$$

and

$$
d(A, B)=0 \Leftrightarrow \bar{A} \equiv \bar{B}
$$

see [11], for example.

We define the set

$$
\bar{Y}=\left\{y \in Y \mid\|y\|_{x} \leq \delta\right\} .
$$

Straightforward application of Theorems 5.3 and 5.5 in [10], using the $C^{1}$-closeness 


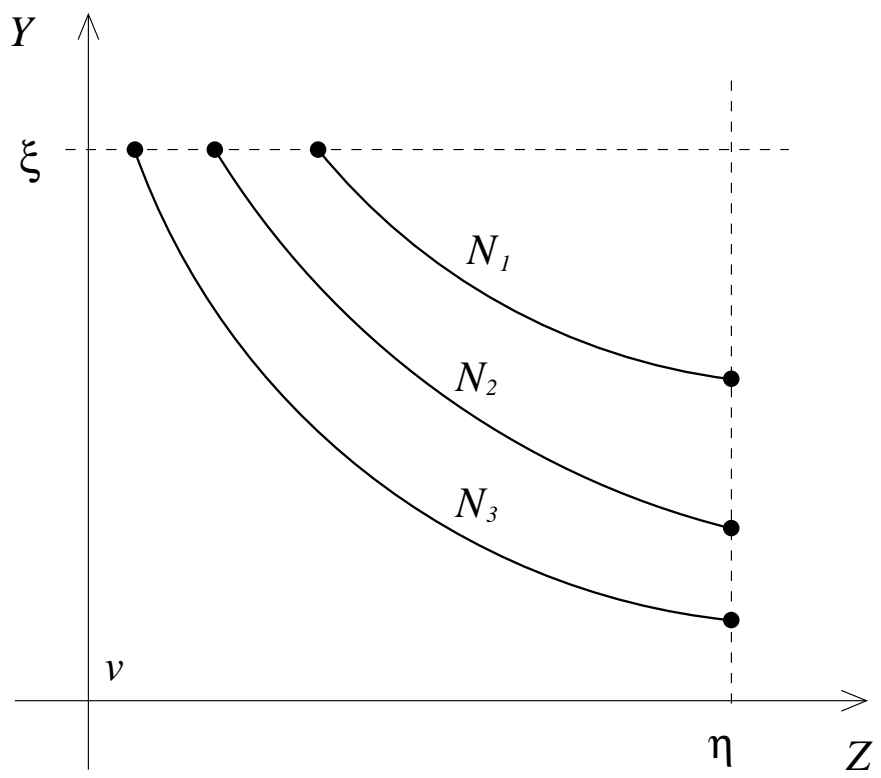

FiguRE 1. Pictorial representation of 3 solutions from Corollary 3.5. Here $0<N_{1}<N_{2}<N_{3}<\infty$

of Proposition 2.4 which holds under Assumption 1.2, yields the following result:

Theorem 3.7. Let Assumption 1.2 hold. Then, for all $\delta$ and $\varepsilon(k, T)$ sufficiently small, there exist functions $\Phi, \Phi_{k} \in C(\bar{Y}, Z)$ such that the mappings $S^{1}$ and $S_{k}^{1}$ have local unstable manifolds

$$
\begin{gathered}
\mathcal{M}=\left\{x \in \mathbb{R}^{m} \mid\|\mathcal{P} x\|_{x} \leq \delta, \mathcal{Q} x=\Phi(\mathcal{P} x)\right\}, \\
\mathcal{M}_{k}=\left\{x \in \mathbb{R}^{m} \mid\|\mathcal{P} x\|_{x} \leq \delta, \mathcal{Q} x=\Phi_{k}(\mathcal{P} x)\right\} .
\end{gathered}
$$

Furthermore, there is a constant $C_{10}>0$ such that $d\left(\mathcal{M}, \mathcal{M}_{k}\right) \leq C_{10} \varepsilon(k, T)$.

Remark 3.8. Although the set $\mathcal{M}_{k}$ is (locally) invariant under $S_{k}^{1}$, it is not locally invariant under $\mathcal{S}_{k}(t)$. To see this, consider the approximation of the equations

$$
\begin{aligned}
p_{t} & =p \\
q_{t} & =-q+p^{2} .
\end{aligned}
$$

It is straightforward to verify that the unstable manifold of the origin for (22) is the set

$$
\mathcal{M}=\left\{(p, q) \in \mathbb{R}^{2} \mid q=\frac{1}{3} p^{2}\right\} .
$$

Hence this is the unstable manifold for $S^{1}$ and it is invariant under $\mathcal{S}(t)$ for any $t \geq 0$. Now, for comparison, consider approximation of (22) by (3) with the Picard splitting $\tilde{f}(v, w)=f(w)$ and $k=1$. Then, for $t \in[0, T]$ and $u=(p, q)^{T}$, we have that $\mathcal{S}_{1}(t) u=v$ where

$$
v=\left((1+t) p,(1-t) q+t p^{2}\right)^{T} .
$$



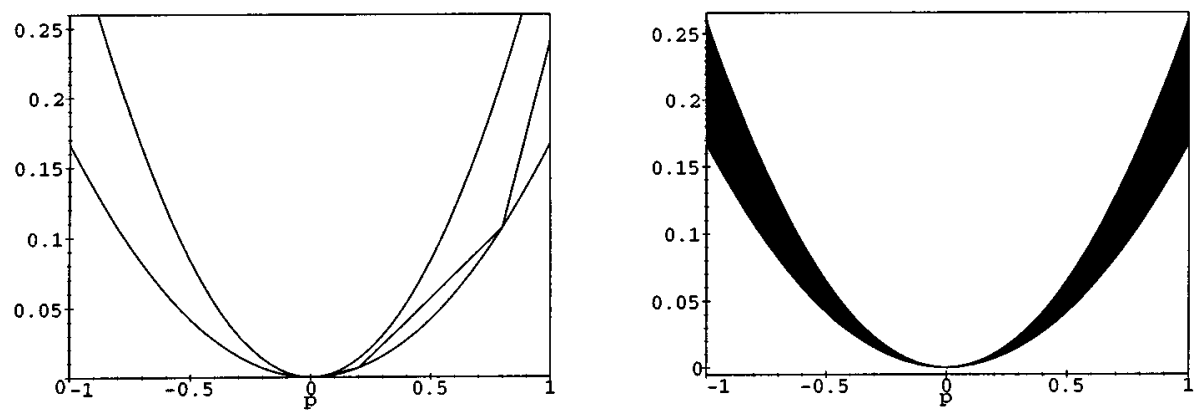

FiguRE 2. Illustrates Remark 3.8

It is straightforward to verify that the set

$$
\mathcal{M}_{1}=\left\{(p, q) \in \mathbb{R}^{2} \mid q=\frac{1}{(3+T)} p^{2}\right\}
$$

is invariant under the map $S_{1}^{1} \equiv \mathcal{S}_{1}(T)$. However, this set is not invariant under $\mathcal{S}_{1}(t)$ in general; indeed, for $t \in[0, T]$,

$$
\mathcal{S}_{1}(t) \mathcal{M}_{1}=\left\{(P, Q) \in \mathbb{R}^{2} \mid Q=\frac{(1+2 t+t T)}{(3+T)(1+t)^{2}} P^{2}\right\} .
$$

This is illustrated in Figure 2. The left figure denotes $\mathcal{M}$ (upper parabola), $\mathcal{M}_{1}$ (lower parabola) and the set $\mathcal{S}_{1}(t) U$ for some $U \in \mathcal{M}_{1}$ (piecewise linear curve). The right figure shows $\bigcup_{t \in[0, T]} \mathcal{S}_{1}(t) \mathcal{M}_{1}$ which clearly differs substantially from $\mathcal{M}_{1}$.

\section{Periodic solutions and attractors}

In the previous section we gave a detailed analysis of the behaviour of the waveform relaxation (3) as an approximation to (2), in the neighbourhood of a hyperbolic equilibrium point. In this section we consider more complicated invariant sets, namely periodic solutions and attractors.

If equation (2) has a hyperbolic periodic solution (that is, all its Floquet multipliers lie off the unit circle except for a simple multiplier at 1 ; see [3]), then the map $S^{1}$ will have a closed invariant curve $\Gamma$. In fact, in the terminology of [5], $S^{1}$ is $r$-normally hyperbolic at $\Gamma$ for some $r \geq 1$. Application of Theorem 4.1 in [5], which requires the $C^{1}$-closeness established in Proposition 2.4 under Assumption 1.2, gives the following result:

Theorem 4.1. Let Assumption 1.2 hold. Assume that (2) has a hyperbolic periodic solution comprising the set $\Gamma$. Then, for all $\varepsilon(k, T)$ sufficiently small, there is a constant $C_{11}>0$ such that $S_{k}^{1}$ has a closed invariant curve $\Gamma_{k}$ satisfying

$$
d\left(\Gamma, \Gamma_{k}\right) \leq C_{11} \varepsilon(k, T) .
$$

Remark 4.2. Although $\Gamma_{k}$ is invariant under $S_{k}^{1}$ it is not, in general, invariant under $\mathcal{S}_{k}(t)$. To illustrate this we consider the equation

$$
z_{t}=\left(i+1-|z|^{2}\right) z
$$

This has the hyperbolic periodic solution $z(t)=e^{i t}$. Now consider again the Picard splitting with $k=1$ so that

$$
\mathcal{S}_{1}(t) z=z\left[\left(1+t-t|z|^{2}\right)+i t\right], \quad t \in[0, T] .
$$


For this example we have

$$
\Gamma=\left\{\left.z \in \mathbb{C}|| z\right|^{2}=1\right\} .
$$

To calculate an invariant curve for $S_{1}^{1}$ set $w(t)=\mathcal{S}_{1}(t) z$ and note that

$$
|w(t)|^{2}=|z|^{2}\left[\left(1+t-t|z|^{2}\right)^{2}+t^{2}\right] .
$$

Thus $|w(T)|^{2}=|z|^{2}$ if

$$
\left(1+T-T|z|^{2}\right)^{2}+T^{2}=1 .
$$

If $T<1$ this gives two values for $|z|$; to define $\Gamma_{1}$ we chose the value which converges to 1 as $T \rightarrow 0$. This gives

$$
\Gamma_{1}=\left\{\left.z \in \mathbb{C}|| z\right|^{2}=\frac{1+T-\left(1-T^{2}\right)^{\frac{1}{2}}}{T}\right\} .
$$

Using (23) we see that, for $t \in[0, T]$,

$\mathcal{S}_{1}(t) \Gamma_{1}=\left\{\left.Z \in \mathbb{C}|| Z\right|^{2}=\frac{1+T-\sqrt{1-T^{2}}}{T}\left(\left[1-\frac{t}{T}\left\{1-\sqrt{1-T^{2}}\right\}\right]^{2}+t^{2}\right)\right\}$.

Thus $\Gamma_{1}$ is not invariant under $\mathcal{S}_{1}(t)$ for $t \in(0, T)$. Figure 3 illustrates this: the left figure shows $\Gamma$ (inner circle) and $\Gamma_{1}$ (outer circle). The right figure shows $\bigcup_{t \in[0, T]} \mathcal{S}_{1}(t) \Gamma_{1}$.

Recall that an attractor is a compact, invariant set which attracts an open neighbourhood of itself. This forms a useful abstraction of the notion of a stable object which is observed after a long period of time in a dynamical system; in particular complicated objects such as the strange attractor for the Lorenz equations, together with simpler objects such as asymptotically stable equilibria and periodic solutions, are included in the definition. We now prove:

Theorem 4.3. Let Assumption 1.1 hold. Suppose that the semigroup generated by (2) has an attractor $\mathcal{A}$. Then $S_{k}^{1}$ has an attractor $\mathcal{A}_{k}$ for all $k$ sufficiently large (resp. $T$ sufficiently small), and

$$
\operatorname{dist}\left(\mathcal{A}_{k}, \mathcal{A}\right) \rightarrow 0
$$

as $k \rightarrow \infty($ resp. $T \rightarrow 0)$.
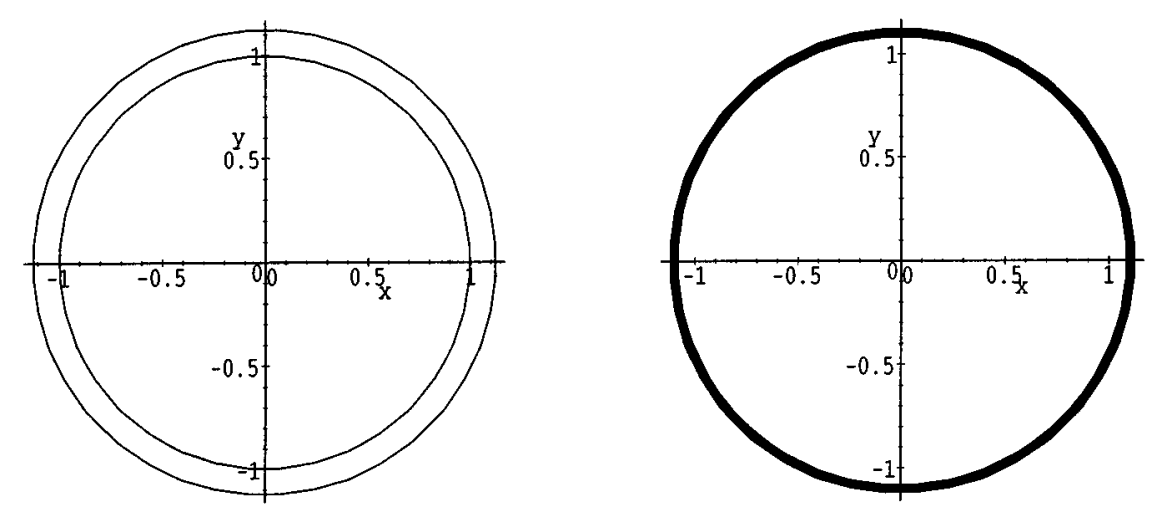

Figure 3. Illustrates Remark 4.2 


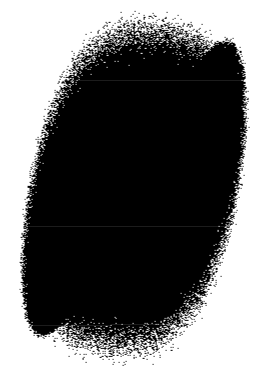

Figure 4. $\mathcal{A}_{1}$, the attractor for $S_{1}^{1}$ applied to the Lorenz equations

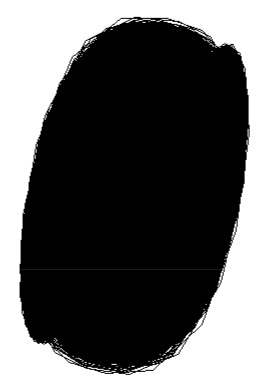

Figure 5. $\mathcal{S}_{1}(t) \mathcal{A}_{1}$; see Remark 4.4

Proof. Since $\mathcal{A}$ is an attractor for the continuous semigroup generated by (2), it is also an attractor for $S^{1}$. Applying Theorem 2.5.4 in [4], noting that (ii) holds in its statement by virtue of Theorem 2.5 , the result follows.

Remark 4.4. As we have seen with the simple invariant sets comprising unstable manifolds and periodic solutions, the invariance of a set $\mathcal{M}$ under $\mathcal{S}_{k}(T)$ does not imply invariance under $\mathcal{S}_{k}(t)$ for $t \in(0, T)$. To illustrate this we consider the approximation of the Lorenz equations

$$
\begin{gathered}
x_{t}=\sigma(y-x), \\
y_{t}=r x-y-x z, \\
z_{t}=x y-b z,
\end{gathered}
$$

by use of the Picard splitting with $k=1$ (Euler's method) and $T=0.02446$. Figures 4 and 5 show solutions of the equations, with parameters set at $\sigma=10, r=$ 28 and $b=\frac{8}{3}$, and initial data $x=0.0, y=1.0, z=0.0$. In both cases the initial transient is not plotted. In Figure 4 the solution is only plotted at integer multiples of $T$ yielding the attractor $\mathcal{A}_{1}$. In Figure 5 we show $\bigcup_{t \in[0, T]} \mathcal{S}_{1}(t) \mathcal{A}_{1}$. Notice the difference between these two objects. 


\section{Conclusion}

Theory and examples have been used to show that waveform relaxation does not form a dynamical system on $\mathbb{R}^{+}$; the numerical examples illustrating this point are somewhat crude, being based on the Picard splitting of $f$ and using only one sweep of the iteration, but they illustrate the underlying principle clearly. We have shown that, if windowed waveform relaxation is to be viewed as a dynamical system, then the map between integer multiples of the window length $T$ is the appropriate approximate dynamical system to compare with the underlying continuous dynamical system. Using this theory we have proved the convergence of a variety of invariant sets of (2) under the approximation (3).

We have chosen not to consider the effect of numerical approximation on the windowed waveform relaxation process because it would complicate the analysis without adding new insight. To include the effect of numerical approximation we would simply need to prove that the numerical approximation of (3) generates a mapping $S_{k, \Delta t}^{1}: \mathbb{R}^{m} \rightarrow \mathbb{R}^{m}$, where $S_{k, \Delta t}^{1} U$ is the numerical approximation to $u_{0}^{(k)}(T)$ with time-step $\Delta t=T / N$, satisfying Proposition 2.4 with $S_{k}^{1}$ and $D S_{k}^{1}$ replaced by $S_{k, \Delta t}^{1}$ and $D S_{k, \Delta t}^{1}$. This requires proving $C^{1}$-convergence of the numerical method over a finite time interval and such a result is given in Theorem 6.2.1 of [11] in the autonomous case; similar techniques can be used in the non-autonomous case considered here.

We have chosen not to give numerical examples where many sweeps of the relaxation are considered simply because the effects are often too small to observe graphically. Our crude numerics amplify the essence of our analysis to a point which is graphically observable. This in no way detracts from our basic point which is that, from a dynamical systems viewpoint, the mapping $S_{k}^{n}$ (resp. its numerical approximation $S_{k, \Delta t}^{n}$ ) is the appropriate object to consider if convergence of invariant sets is to be studied for large $k$ (resp. large $k$ and small $\Delta t$ ).

\section{REFERENCES}

1. Alfredo Bellen and Marino Zennaro, The use of Runge-Kutta formulae in waveform relaxation methods, Appl. Numer. Math. 11 (1993), 95-114. MR 94c:65090

2. Morten Bjørhus, A note on the convergence of discretized dynamic iteration, BIT 35 (1995), 291-296.

3. J. K. Hale, Ordinary differential equations, Wiley, Chichester, 1969. MR 54:7918

4. - Asymptotic behaviour of dissipative systems, Mathematical surveys and Monographs, no. 25, AMS, Providence, Rhode Island, 1988. MR 89g:58059

5. M. Hirsch, C. Pugh, and M. Shub, Invariant manifolds, Springer-Verlag, New York, 1977. MR 58:18595

6. Arieh Iserles, Stability and dynamics of numerical methods for nonlinear ordinary differential equations, IMA J. Num. Anal. 10 (1990), 1-30. MR 91e:65105

7. Ekachai Lelarasmee, Albert E. Ruehli, and Alberto L. Sangiovanni-Vincentelli, The waveform relaxation method for time-domain analysis of large scale integrated circuits, IEEE Trans. on CAD of IC and Syst. 1 (1982), 131-145.

8. Ulla Miekkala and Olavi Nevanlinna, Convergence of dynamic iteration methods for initial value problems, SIAM J. Sci. Stat. Comput. 8 (1987), 459-482. MR 89f:65076

9. Olavi Nevanlinna, Remarks on Picard-Lindelöf iteration, BIT 29 (1989), 328-346, 535-562. MR 91b:65088

10. Andrew M. Stuart, Perturbation theory for infinite dimensional dynamical systems, Advances in Numerical Analysis (M. Ainsworth, J. Levesley, W.A. Light, and M. Marletta, eds.), Oxford University Press, 1995. CMP 96:05 
11. Andrew M. Stuart and A. R. Humphries, Dynamical systems and numerical analysis, Cambridge University Press, Cambridge, 1996. CMP 96:16

Department of Mathematical Sciences, The Norwegian Institute of Technology, N7034 Trondheim, NorWay

Current address: Forsvarets Forskningsinstitutt, PO Box 25, N-2007 Kjeller, Norway

E-mail address: mbj@ffi.no

Department of Mechanical Engineering, Division of Mechanics and Computation, Stanford University, Durand Bullding, Room 257, Stanford, California 94305

E-mail address: stuart@sccm.stanford.edu 